\title{
Face Localization using an Effective Co-Evolutionary Genetic Algorithm
}

\author{
Farshid Hajati ${ }^{1,2}$, Caro Lucas ${ }^{3}$ \\ ${ }^{1}$ Faculty of Electrical Eng., Amirkabir University of \\ Technology, Tehran, Iran \\ ${ }^{2}$ School of Engineering, Griffith University, QLD 4111, \\ Australia \\ ${ }^{3}$ School of Electrical and Computer Eng., University of \\ Tehran, Tehran, Iran \\ hajati@aut.ac.ir, lucas@ipm.ir
}

\author{
Yongsheng $\mathrm{Gao}^{2,4}$ \\ ${ }^{2}$ School of Engineering, Griffith University, QLD 4111, \\ Australia \\ ${ }^{4}$ Queensland Research Lab, National ICT Australia, Australia \\ yongsheng.gao@griffith.edu.au
}

\begin{abstract}
In this paper, a new method for face localization in color images, which is based on co-evolutionary systems, is introduced. The proposed method uses a co-evolutionary system to locate the eyes in a face image. The used coevolutionary system involves two genetic algorithm models. The first GA model searches for a solution in the given environment, and the second GA model searches for useful genetic information in the first GA model. In the next step, by using the location of eyes in image the parameters of face's bounding ellipse (center, orientation, major and minor axis) are computed. To evaluate and compare the proposed method with other methods, high order Pseudo Zernike Moments (PZM) are utilized to produce feature vectors and a Radial Basis Function (RBF) neural network is used as the classifier. Simulation results indicate that the speed and accuracy of the new system using the proposed face localization method which uses a co-evolutionary approach is higher than the system proposed in $[10]$.
\end{abstract}

Keywords-face localization; genetic algorithm; co-
evolutionary

\section{INTRODUCTION}

Automatic face recognition has been an active research topic due to its extensive range of applications, such as access control systems, criminal identification and authentication in secure systems [1]. A face recognition system generally consists of three steps: face localization, feature extraction and classification. In the first step, localization of the face region from $2 \mathrm{D}$ images through segmentation is carried out. This step is very challenging due to unknown position, orientation and scaling of faces in an arbitrary image [2, 3, 4]. The second step involves determination of pertinent feature vector from the localized image [5], while the third step prepares classification of the face image based on the information created from the feature vectors $[5,6]$.

As mentioned, face region segmentation or face localization is a fundamental step in the process of face recognition. The accuracy of the localized face's ellipse has a heavy influence on the recognition performance [7]. Genetic algorithms (GA's) are optimization techniques based on the mechanism of natural selection [8]. Recently, GA's have been widely used as a tool in pattern recognition applications. Because of their problems in convergence, in this paper we use co-evolutionary GA's for face localization. In co-evolutionary systems, by contrast, the fitness of an individual depends on both how well it solves a problem and on the fitness of other individuals [9].

At first, the location of eyes in the face image is extracted by using a co-evolutionary system. Then, by using the location of eyes in the face image, the parameters of face's bounding ellipse (center, orientation, major and minor axis) are computed and the face's ellipse is extracted. Next, for evaluating of the proposed system we use Pseudo Zernike Moments (PZM) to generate the feature vector elements and an RBF neural network with the structure described in [10] as the classifier. Experiments show that the accuracy and speed of the proposed method are higher than the system proposed in [10].

The organization of this paper is as follows: Section 2 presents the face localization method. Feature extraction and classifier design are described in Section 3 and 4, and finally, Sections 5 and 6 present the experimental results and conclusion.

\section{FACE LOCALIZATION}

have been proposed for face localization and detection. A critical survey on face localization and detection can be found in reference [11]. To ensure a robust and accurate feature extraction that distinguishes between different individuals effectively, the location of the face region should be determined as accurate as possible. In this paper, we have proposed a new method for face localization in color images which uses the co-evolutionary GA's to locate the eyes and compute and extract the face's ellipse. The proposed face localization method consists of three steps: first, skin filter step which detects the skin area in a face image; second, eyes localization step in which the location of eyes in the face skin region will be detected; and finally, face's ellipse extraction step in which the face's bounding ellipse is extracted. The more detailed description of these steps is presented in the following: 


\section{A. Skin Filter}

In face images many information such as background and hairs are redundant for eye detection. So, we first detect the skin area of faces by the skin filter. Also, the appearance of the skin color depends on the lighting conditions; therefore, we use a lighting compensation technique introduced in [12] to normalize the color appearance in face images. In this technique we regard pixels with the top 5 percent of the luma (nonlinear gamma-corrected luminance) values in the image as the "reference white". The $R, G$ and $B$ components of a face color image are adjusted so that the average gray values of these reference-white pixels are equal to 255 .

The color of face's skin of human is composed of a combination of blood (red) and melanin (yellow, brown). Therefore, human's face skin has a restricted range of hues and is somewhat saturated. The skin filter is based on the Fleck and Forsyth algorithm [13]. The skin filter starts by transforming the input $R, G$ and $B$ values into log-opponent values:

$$
\begin{gathered}
I=[L(R)+L(G)+L(B)] / 3 \\
R_{g}=L(R)-L(G) \\
B_{y}=L(B)-[L(G)+L(R)] / 2
\end{gathered}
$$

where $L(X)=105 \log (X+1)$.

In the $\log$ transformation, 105 is a convenient scaling constant. The log transformation makes the $R_{g}$ and $B_{y}$ values intensity independent. Next, smoothed texture and color planes are extracted. The $R_{g}$ and $B_{y}$ arrays are smoothed with a median filter. To compute texture amplitude, the intensity image is smoothed with a median filter, and the result is subtracted from the original image. The absolute values of these differences are run through a second median filter. The texture amplitude and the smoothed $R_{g}$ and $B_{y}$ values are then passed to a tightly-tuned skin filter. It marks as probable skin all pixels whose texture amplitude is small, and whose hue and saturation values are appropriate. Hue and saturation are simply the direction and magnitude of the vector $\left(R_{g}, B_{y}\right)$.

Because, the color of facial features such as: eyes, eyebrow and noise is not similar to skin color, these areas will not pass the tightly-tuned skin filter, creating holes in skin region. Therefore, the output of the initial skin filter is expanded by some morphological operations to include these regions. Fig. 1 shows an input image and its output for skin filter. Now, by means of this segmented color image we can limit the localization process of eyes in the face region and remove redundant information such as background and hairs.

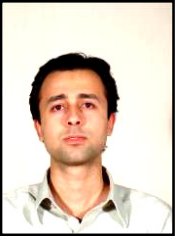

(a)

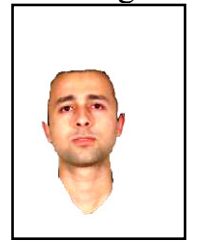

(b)
Figure 1. (a) An input image for skin filter, (b) the output of the skin filter.

\section{B. Eyes Localization}

Among the various facial features, eyes are the most prominent features for recognition and estimation of head pose [14]. Most approaches for eye detection in gray scale images are template-based [15], [16]. In these approaches a template of eye is moved over the image and at each place the correlation between the template and subimage is computed. Finally, a place in which amplitude of the correlation is maximum, is selected as the eye location. Because of the large number of data and long time of process, this method is not appropriate for color images. For this reason, we proposed a new method to locate the eyes which uses co-evolutionary systems. A co-evolutionary system is adopted in our work to solve the most troubles of traditional methods [9]. Due to its good reputation in the application of searching and optimization, good template matching performance (in term of speed and matching error) can be expected.

The used co-evolutionary system involves two genetic algorithm models [17]. The first GA model searches for a solution in a given environment, and the second GA model searches for useful genetic information in the first GA model. The resulted system has high search ability due to the co-evolution consisting of these two GA models. As depicted in Fig. 2, the first GA model (GA-1) is the traditional GA model, storing individuals that may be "transcripted" by the genetic information which is discovered by the second GA model (GA-2). On the other hand, the GA-2, searches for useful genetic information on the GA-1, and the fitness value of each individual of the GA-2 is calculated by referring to the population distribution of the individuals in the GA-1.

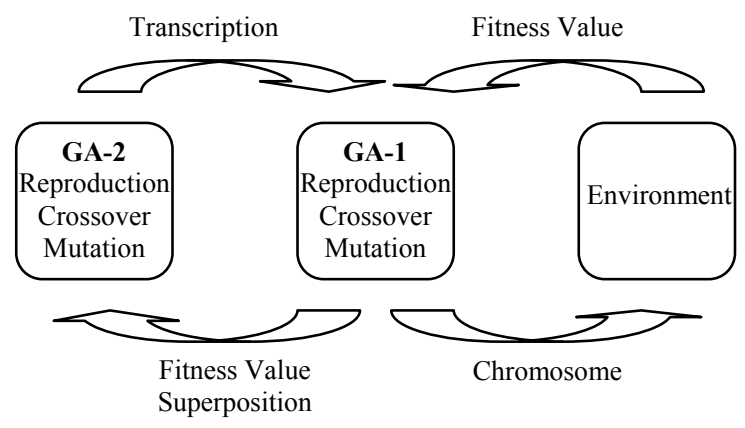

Figure 2. The used co-evolutionary system.

The GA-1 is the traditional GA model that searches for a good individual fit to the given environment. Each chromosome in this environment has an associated fitness value that is defined as

$$
\begin{gathered}
f_{i}=R_{i}^{r}+R_{i}^{l}+\sigma \cdot \operatorname{Cos}\left(\theta_{i}\right) \\
\theta_{i}=\tan ^{-1}\left(\left(y_{l}-y_{r}\right) /\left(x_{l}-x_{r}\right)\right)
\end{gathered}
$$

where $R_{i}^{r}$ and $R_{i}^{l}$ are correlation values between candidate 
eye regions and right and left eye templates, respectively. Scaling parameter $0<\sigma<1$ is selected experimentally and $\theta_{i}$ is the angle between the line joining the eyes and the horizontal line ( $\theta$ in Fig. 5). Because the face images we used for face recognition were frontal and upright images, to eliminate the candidate eyes which are not horizontal, the third term in (4) is added.

Because each eye in the subject image is localized by its coordinates ( $\mathrm{x}$ and $\mathrm{y}$ ), each chromosome in the population during the evolutionary search has four genes: the coordinates of the left eye $\left(\mathrm{x}_{1}, \mathrm{y}_{\mathrm{l}}\right)$ and the coordinates of right eye $\left(\mathrm{x}_{\mathrm{r}}, \mathrm{y}_{\mathrm{r}}\right)$. Chromosomes are coded in binary form as shown in Fig. 3. In this form the length of each chromosome is 32 .

\begin{tabular}{|c|c|c|c|}
\hline $\mathrm{x}_{\mathrm{r}}-8$ bits & $\mathrm{y}_{\mathrm{r}}-8$ bits & $\mathrm{x}_{\mathrm{l}}-8$ bits & $\mathrm{y}_{\mathrm{l}}-8$ bits \\
\hline
\end{tabular}

Figure 3. The chromosome for localization of eyes.

To improve search ability of GA-1, GA-2 is adopted to inform GA-1 the candidate subspaces to be searched for. The GA-2 individuals consist of 0's, 1's and *'s representing genetic information in GA-1. The "superposition" operator uses genetic information in the GA-1 to calculate the fitness values of GA-2 individuals, and the "transcription" operator propagates effective genetic information in GA-2 into GA-1 population. These two operators are shown in Fig. 4.

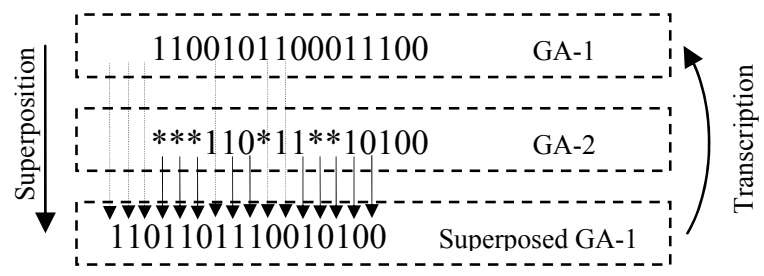

Figure 4. Mechanism of superposition and transcription operators.

GA-2 searches for useful genetic information in GA-1. Then, the fitness value of $j$-th individual of GA-2, $F_{j}$, is described as follows:

The superposition operation of each GA-2 onto GA-1 is carried out $n$ times.

(1) First, it is randomly decided which GA-1 individuals are superposed by GA-2 individual $j$.

(2) GA-1 individuals that are superposed by GA-2 individual $j$ are denoted as $i_{1}, \ldots, i_{n}$. And the resultant superposed GA-1 individuals are denoted by $\tilde{i}_{1}, \ldots, \tilde{i}_{n}$.

(3) To calculate the fitness value of GA-2 individual $j$, the fitness evaluation of each of the superposition operations is defined as the contribution of the superposition operation to GA-1 individual defined by the following equation:

$$
\tilde{f}_{k}=\max \left(0, f_{\tilde{i}_{k}}-f_{i_{k}}\right) \quad k=1, \ldots, n
$$

Where, $f_{i_{k}}$ denotes the fitness value of the $i_{k}$ individual in the GA-1.

(4) Finally, the fitness $F_{j}$ of GA-2 individual $j$ is given by the following equation:

$$
F_{j}=\sum_{k=1}^{n} \tilde{f}_{k}
$$

A superposed chromosome is used to calculate the fitness value of GA-2 individuals. The probability of applying the transcription operation is defined as:

$$
P=\left\{\begin{array}{lr}
\tilde{f}_{k} /\left(f_{\max }-f_{\min }\right) & \tilde{f}_{k}>0 \\
0 & \text { other }
\end{array}\right.
$$

where $f_{\max }$ and $f_{\min }$ denote maximum and minimum fitness values in GA-1, respectively, and hence $P$ is a value such that $0<\mathrm{P}<1$.

By applying this optimization system to the face region extracted in previous step, the position of eyes in the face image is found precisely. Now, by using the location of eyes we can extract the face's bounding ellipse in the next step.

\section{Face's Ellipse Extraction}

In face localization methods, it is well known that faces are characterized by an elliptical shape and an ellipse can approximate the shape of a face [7]. An ellipse is exactly defined by its center $\left(x_{0}, y_{0}\right)$, its orientation $\theta$ and the length $\alpha$ and $\beta$ of its minor and major axis, respectively. In this paper, the parameters of face's bounding ellipse are computed by using the location of eyes in the face region. Experimental results show that the center of face's ellipse in each person is located on the perpendicular bisector of the line joining the eyes, $0.43 d$ ( $d$ is the distance between the eyes) below the mentioned line. Therefore, the following relations for computing the parameters of face's ellipse hold (Fig. 5):

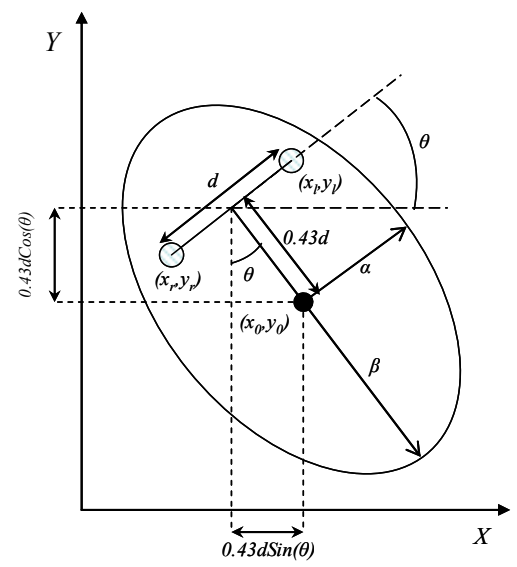

Figure 5. Computation of face's ellipse parameters. 


$$
\begin{gathered}
\theta=\tan ^{-1}\left(\left(y_{l}-y_{r}\right) /\left(x_{l}-x_{r}\right)\right) \\
x_{0}=\left(x_{l}+x_{r}\right) / 2+0.43 \times d \times \operatorname{Sin}(\theta) \\
y_{0}=\left(y_{l}+y_{r}\right) / 2-0.43 \times d \times \operatorname{Cos}(\theta) \\
\beta=1.3 \times d \\
\alpha=0.9 \times d
\end{gathered}
$$

where $\left(x_{l}, y_{l}\right),\left(x_{r}, y_{r}\right)$ and $d=\left(\left(x_{l}-x_{r}\right) 2+\left(y_{l}-y_{r}\right)^{2}\right)^{1 / 2}$ indicate the coordinates of left eye and right eye and the distance between the eyes, respectively. Now, by means of these parameters we can accurately extract the face's ellipse.

\section{FEATURE EXTRACTION}

For feature extraction step, we use Pseudo Zernike Moments. The advantages of these moments are that they are shift, rotation and scale invariant and very robust in the presence of noise [7]. Pseudo Zernike polynomials are orthogonal sets of complex-valued polynomials defined as:

$$
V_{n m}(x, y)=R_{n m}(x, y) \exp \left(j m \tan ^{-1}\left(\frac{y}{x}\right)\right)
$$

where $x^{2}+y^{2} \leq 1, n \geq 0,|m| \leq n$ and radial polynomials, $R_{n m}$, are defined as:

$$
R_{n m}(x, y)=\sum_{s=0}^{n-|m|} D_{n,|m|, s}\left(x^{2}+y^{2}\right)^{\frac{n-s}{2}}
$$

where

$$
D_{n,|m|, s}=(-1)^{s} \frac{(2 n+1-s) !}{s !(n-|m|-s) !(n-|m|-s+1) !}
$$

The PZM of order $n$ and repetition $m$ can be computed using the scale invariant central moments $C M_{p, q}$ and the radial geometric moments $R M_{p, q}$ as follows:

$$
\begin{aligned}
P Z M_{n m}= & \frac{n+1}{\pi} \sum_{(n-m-s) \text { even, } s=0}^{n-|m|} D_{n,|m|, s} \sum_{a=0}^{k} \sum_{b=0}^{m}\left(\begin{array}{l}
k \\
a
\end{array}\right)\left(\begin{array}{l}
m \\
b
\end{array}\right) \\
& (-j)^{b} C M_{2 k+m-2 a-b, 2 a+b} \\
& +\frac{n+1}{\pi} \sum_{(n-m-s)}^{n-|m|} D_{n,|m|, s} \sum_{a=0}^{d} \sum^{m}\left(\begin{array}{l}
d \\
a
\end{array}\right)\left(\begin{array}{l}
m \\
b
\end{array}\right) \\
& (-j)^{b} R M_{2 d+m-2 a-b, 2 a+b}
\end{aligned}
$$

where $k=(n-s-m) / 2, d=(n-s-m-1) / 2$ and also $C M_{p, q}$ and $R M_{p, q}$ are defined as follows:

$$
\begin{gathered}
C M_{p, q}=\frac{\mu_{p, q}}{M_{00}{ }^{(p+q+2) / 2}} \\
R M_{p, q}=\frac{\sum_{x} \sum_{y} f(x, y)\left(\hat{x}^{2}+\hat{y}^{2}\right)^{1 / 2} \hat{x} \hat{y}}{M_{00}{ }^{(p+q+2) / 2}}
\end{gathered}
$$

where $x=x-x_{0}, y=y-y_{0}$ and $x_{0}, y_{0}, M_{p q}$ and $\mu_{p q}$ are defined as follows:

$$
\begin{gathered}
x_{0}=\frac{m_{10}}{m_{00}}, y_{0}=\frac{m_{01}}{m_{00}} \\
M_{p q}=\sum_{x} \sum_{y} f(x, y) x^{p} y^{q} \\
\mu_{p q}=\sum_{x} \sum_{y} f(x, y)\left(x-x_{0}\right)^{p}\left(y-y_{0}\right)^{q}
\end{gathered}
$$

where $M_{p q}$ and $\mu_{p q}$ are geometrical and central moments of the 2D image $(f(x, y))$, respectively.

\section{Classifier Design}

Radial Basis Function (RBF) neural networks have found to be very attractive for many engineering problems, because: (1) they are universal approximators, (2) they have a very compact topology, (3) their learning speed is very high because of their locally tuned neurons [5]. The RBF neural network has a feed forward architecture with an input layer, a hidden layer and an output layer. In this paper, an RBF neural network is used as the classifier in the face recognition system and the inputs to this network are feature vectors derived from the feature extraction method described in the previous section.

For this purpose, we use an RBF neural network with the structure presented in [10] for classification of feature vectors. This structure is depicted in Fig. 6.

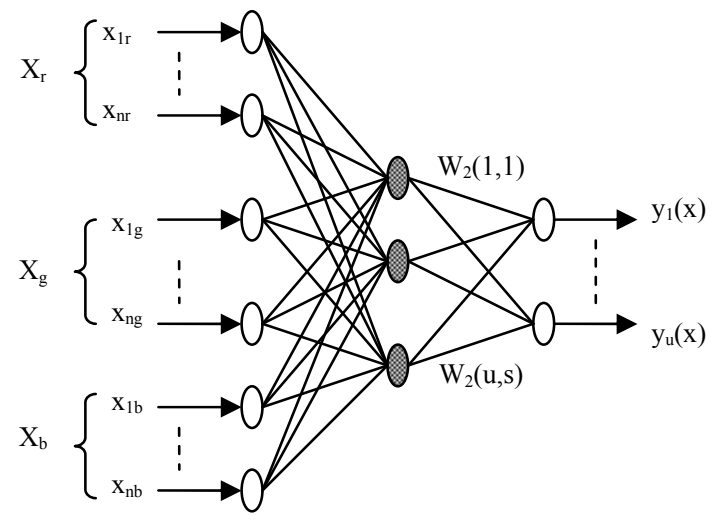

Figure 6. The structure of the used RBF neural network

In this structure three feature vectors corresponding to $R$, $G$ and $B$ components are fed to the RBF units in a parallel form. In this case, the distance function of RBF units is as follows:

$$
D_{i}(X)=\frac{\left\|X_{r}-C_{i r}\right\| .\left\|X_{g}-C_{i g}\right\| .\left\|X_{b}-C_{i b}\right\|}{\sigma_{i}}
$$

where $X_{r}, X_{g}$ and $X_{b}$ represent the n-dimensional input feature vectors of $R, G$ and $B$ components, respectively. $C_{i r}$, $C_{i g}$ and $C_{i b}$ are n-dimensional vectors called the centers of $R$, $G$ and $B$ components of $i$-th RBF unit. $\sigma_{i}$ is the width of $i$-th $\mathrm{RBF}$ unit and is selected as: 


$$
\sigma_{i}=\alpha \min \left\{\left\|C_{i r}-C_{j r}\right\| .\left\|C_{i g}-C_{j g}\right\|\left\|C_{i b}-C_{j b}\right\|\right\} \quad i \neq j
$$

where the parameter $0<\alpha \leq 1$ has to be selected experimentally.

We set the number of input nodes in the input layer of neural network equal to the total number of feature vector elements extracted for the three color components. The number of nodes in the output layer is set equal to the number of face classes. In this structure, the RBF unit parameters and output connection weights are determined using the procedures presented in [10].

\section{EXPERIMENTAL RESULTS}

To evaluate the algorithm, we used a database which we gathered from Persian faces. Since our system is going to be practically used for recognition purposes in our laboratory, this database which is a representative of Persian faces would lead to better results. Experimental studies carried out on our database including the frontal color images of 500 faces from 100 individuals with different facial expressions, have been used to evaluate the performance of the proposed method. In this database each person has changed his/her face expression in each of the 5 samples. Some examples of our database images are shown in Fig. 7.

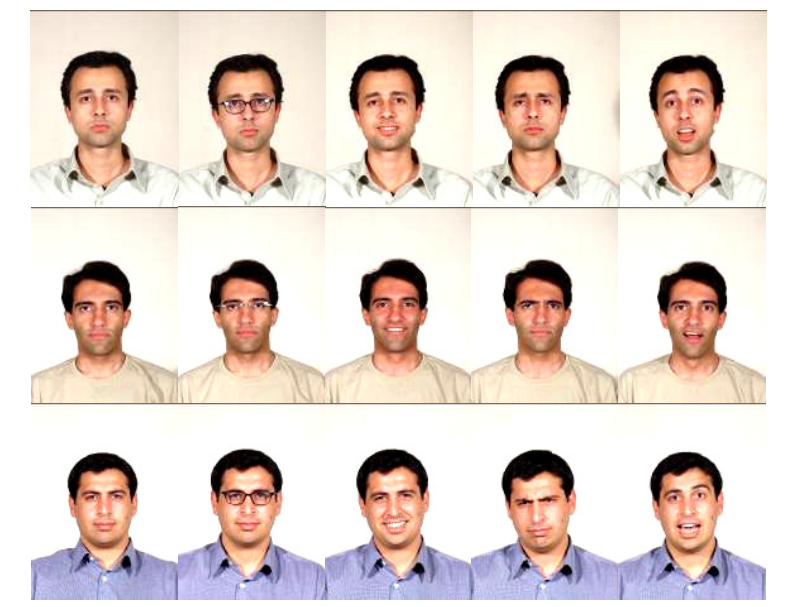

Figure 7. Some examples of prepared database showing different facial expressions

After applying the face localization method that is described in section 2, we have face images in which the redundant information such as background and hair are omitted. Fig. 8 shows the localized face images after using the proposed face localization system.

In the feature extraction step, because of PZM advantages [7], we extract PZM of localized face images with different orders and different element numbers. Simulation results (Fig. 9) show that the classification error rate using PZM with the order of 9 or higher is lower than the other orders.

In this paper, we define three categories for feature vectors based on the order (n) of the PZM which are shown in Table I. Our experimental study indicates that this categorization process allows a good discrimination capability with a lower dimension feature vector.

Simulation has been done in three steps based on the order (n) of PZM as described in Table I. A total of 300 images are used to train and another 200 are used to test (3 images for training and 2 images for testing, for each of the 100 individuals). The neural network classifier is trained in each category based on training images. The outcome of experimental results is shown in the Table II.

This table shows that the third category $(n=9, \ldots, 12)$ is more appropriate for classification of faces within the proposed system. To compare this system with the system presented in [10], the classification error and classification time of the two systems are listed in Table III. These experiments have been carried out by using a Pentium 4 processor, in the Matlab (version 7.2) environment. The simulation results presented in Table III show that the proposed system is faster and more accurate than the older system.

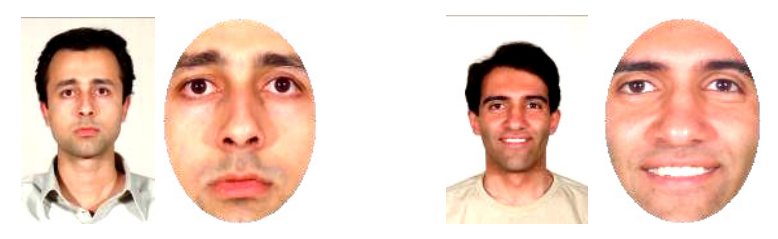

Figure 8. The results of face localization method

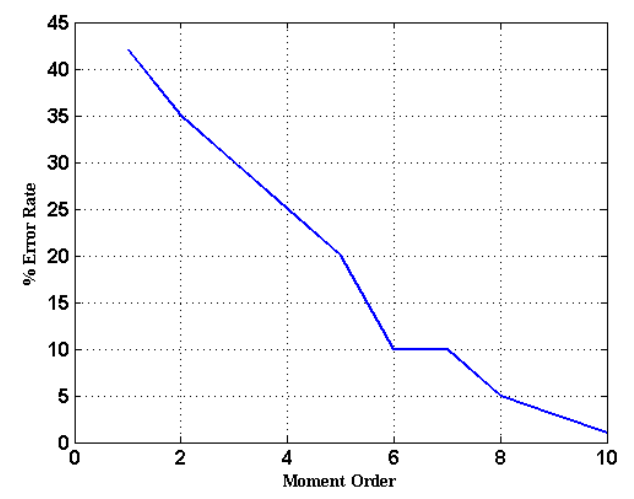

Figure 9. Classification error rate for different order of PZM

TABLE I. FEATURE VECTOR ELEMENTS BASED ON PZM

\begin{tabular}{|c|ll|}
\hline Category no. & & \multicolumn{1}{|c|}{ PZM feature elements } \\
\hline & $\mathrm{n}=0$ & $\mathrm{~m}=0$ \\
& $\mathrm{n}=1$ & $\mathrm{~m}=0,1$ \\
& $\mathrm{n}=2$ & $\mathrm{~m}=0,1,2$ \\
1 & $\mathrm{n}=3$ & $\mathrm{~m}=0,1,2,3$ \\
& $\mathrm{n}=4$ & $\mathrm{~m}=0,1,2,3,4$ \\
& $\mathrm{n}=5$ & $\mathrm{~m}=0,1,2,3,4,5$ \\
& $\mathrm{n}=6$ & $\mathrm{~m}=0,1,2,3,4,5,6$ \\
& $\mathrm{n}=7$ & $\mathrm{~m}=0,1,2,3,4,5,6,7$ \\
& $\mathrm{n}=8$ & $\mathrm{~m}=0,1,2,3,4,5,6,7,8$ \\
\hline
\end{tabular}




\begin{tabular}{|l|ll|}
\hline & $\mathrm{n}=6$ & $\mathrm{~m}=0,1,2,3,4,5,6$ \\
$\mathrm{n}=7$ & $\mathrm{~m}=0,1,2,3,4,5,6,7$ \\
& $\mathrm{n}=8$ & $\mathrm{~m}=0,1,2,3,4,5,6,7,8$ \\
& $\mathrm{n}=9$ & $\mathrm{~m}=0,1,2,3,4,5,6,7,8,9$ \\
& $\mathrm{n}=10$ & $\mathrm{~m}=0,1,2,3,4,5,6,7,8,9,10$ \\
\hline & $\mathrm{n}=9$ & $\mathrm{~m}=0,1,2,3,4,5,6,7,8,9$ \\
& $\mathrm{n}=10$ & $\mathrm{~m}=0,1,2,3,4,5,6,7,8,9,10$ \\
& $\mathrm{n}=11$ & $\mathrm{~m}=0,1,2,3,4,5,6,7,8,9,10,11$ \\
& $\mathrm{n}=12$ & $\mathrm{~m}=0,1,2,3,4,5,6,7,8,9,10,11,12$ \\
\hline
\end{tabular}

TABLE II. EXPERIMENTAL RESULTS

\begin{tabular}{|c|c|c|c|}
\hline \multicolumn{2}{|c|}{ Feature vectors } & \multicolumn{2}{c|}{ Testing phase } \\
\hline $\begin{array}{c}\text { Category } \\
\text { no. }\end{array}$ & NFE $^{1}$ & $\mathrm{NM}^{2}$ & $\mathrm{ER}^{3}$ \\
\hline 1 & 45 & 13 & $6.5 \%$ \\
\hline 2 & 45 & 5 & $2.5 \%$ \\
\hline 3 & 46 & 2 & $1 \%$ \\
\hline
\end{tabular}
${ }^{2}$ Number of Feature Element for each component
${ }^{3}$ Eumber of Misclassification Rate = NM/Number of total testing images

TABLE III. COMPARISON OF TWO SYSTEMS

\begin{tabular}{|c|c|c|c|c|c|}
\hline \multicolumn{2}{|c|}{ Feature vectors } & \multicolumn{2}{c|}{ Classification error } & \multicolumn{2}{c|}{ Classification time } \\
\hline $\begin{array}{c}\text { Category } \\
\text { no. }\end{array}$ & NFE & $\begin{array}{c}\text { Proposed } \\
\text { method }\end{array}$ & $\begin{array}{c}\text { Ref. } \\
{[10]}\end{array}$ & $\begin{array}{c}\text { Proposed } \\
\text { method }\end{array}$ & $\begin{array}{c}\text { Ref. } \\
{[10]}\end{array}$ \\
\hline 1 & 45 & $6.5 \%$ & $7 \%$ & $15.5 \mathrm{~s}$ & $20.1 \mathrm{~s}$ \\
\hline 2 & 45 & $2.5 \%$ & $3 \%$ & $20.1 \mathrm{~s}$ & $24.6 \mathrm{~s}$ \\
\hline 3 & 46 & $1 \%$ & $1.2 \%$ & $28.3 \mathrm{~s}$ & $33 \mathrm{~s}$ \\
\hline
\end{tabular}

\section{CONCLUSION}

In this paper, a new method for face localization in color images is presented. This new method uses a coevolutionary approach to locate the eyes in face images. This co-evolutionary system consists of two traditional genetic algorithms. The first GA model searches for a solution in a given environment, and the second GA model searches for useful genetic information in the first GA model. In the next step, by using the location of eyes, the parameters of the face's bounding ellipse (center, orientation, major and minor axis) are computed and the face's ellipse is extracted. To evaluate the proposed method the PZMs are extracted and an RBF neural network is used for classification. The experimental results show that the proposed system is faster and more accurate than our previous system.

\section{ACKNOWLEDGMENT}

The author would like to bless Prof. Caro Lucas to be calm in the heaven with his God.

\section{REFERENCES}

[1] R. Chellappa, C. L. Wilson, and S. Sirohry, "Human and machine recognition of faces: a survey," Proceedings of the IEEE, vol. 83, no. 5, pp. 705-740, 1995.

[2] G. Yang and T. S. Huang, "Human face detection in a complex background," Journal of Pattern Recognition, vol. 27, no. 1, pp. 53-63, 1994.

[3] J. Haddadnia and K. Faez, "Human face recognition using radial basis function neural network," Third Int'l Conf. on Human and Computer, pp. 137-142, 2000.

[4] K. Sobotta and I. Pitas, "Face localization and facial feature extraction based on shape and color information," IEEE Int'l Conf. on Image Processing, pp. 483-486, 1996.

[5] J. Haddadnia and K. Faez, "Neural network human face recognition based on moment invariants," Proceeding of IEEE International Conference on Image, pp. 1018-1021, 2001.

[6] H. A. Rowley, S. Zbaluja, and T. Kanade, "Neural network based face recognition," IEEE Trans. on Pattern Recognition and Machine Intelligence, vol. 20, no. 1, pp. 23-28, 1998.

[7] H. Haddadnia, K. Faez, and M. Ahmadi, "An efficient human face recognition system using pseudo Zernike moment invariant and radial basis function neural network," Int'l Journal of Pattern Recognition and Artificial Intelligence, vol. 17, no. 1, pp.41-62, 2003.

[8] M. Srinivas and L. M. Patnik, "Genetic algorithms: a survey," Int'1 Journal on Computer Vision, vol. 27, no. 6, pp. 17-26, 1994.

[9] J. Morrison and F. Oppacher, "A general model of coevolution for genetic algorithms," Proceeding of Fourth Int'l Conf. on Artificial Neural Network \& Genetic Algorithms, pp. 314-323, 1997.

[10] F. Hajati, K. Faez and S.K. Pakazad, "An efficient method for face localization and recognition in color images," Proceeding of IEEE Int'l Conf. on Systems, Man, and Cybernetics, pp. 4214-4219, 2006.

[11] J. Daugman, "Face detection: a Survey," Journal of Computer Vision and Image Understanding, vol. 83, no. 3, pp. 236-274, 2001.

[12] R.L. Hsu, M.A. Mottalab, and A.K. Jain, "Face detection in color images," IEEE Transaction on Pattern Analysis and Machine Intelligence, vol. 24, no. 5, pp. 696-706, 2002.

[13] M. Fleck and D. Forsyth, "Automatic detection of human nudes," International Journal of Computer Vision, vol. 32, no. 1, pp. 63-77, 1999.

[14] A. Nikolaidis and I. Pitas, "Facial feature extraction and pose determination," Pattern Recognition, vol. 33, no. 11, pp. 1783-1791, 2000.

[15] W. Huang, Q. Sun, C.P. Lam, and J.K. Wu, "A robust approach to face and eye detection from images with cluttered background," Proc. of Int'l Conf. on Pattern Recognition, pp. 1783-1791, 2000.

[16] K. M. Lam and H. Yan, "Locating and extracting the eye in human face images," Journal of Pattern Recognition, vol. 20, no. 5, pp. 771-779, 1996.

[17] H. Handa, N. Baba, and O. Katai, "Genetic algorithm involving co-evolutionary mechanism to search for effective genetic information," Proceeding of Int'l Conf. on Evolutionary Computation, pp.709-71, 1997. 